\title{
Complete Molar Pregnancy in Postmenopausal Woman
}

\author{
Ahmed Samy El-Agwany ${ }^{1} \cdot$ Helmy Abdelsattar $\operatorname{Rady}^{1} \cdot$ Hassan Mansour Hegab $^{1}$
}

Received: 11 April 2016/Accepted: 1 December 2016/Published online: 19 December 2016

(C) Indian Association of Surgical Oncology 2016

\begin{abstract}
Gestational trophoblastic disease spectrum ranges from benign hydatidiform mole to trophoblastic malignancy (placental site trophoblastic tumor and choriocarcinoma). Benign gestational trophoblastic disease occurs in women of reproductive age and is extremely rare in postmenopausal women. We describe a case of complete vesicular mole in a 55-year-old postmenopausal woman complaining of postmenopausal bleeding. To our knowledge, our case represents one of the rare descriptions in the world literature of a benign complete hydatidiform mole in a postmenopausal woman.
\end{abstract}

Keywords Vesicular mole $\cdot$ Menopause $\cdot$ Bleeding $\cdot$ Case report $\cdot$ Pregnancy

\section{Introduction}

Vesicular mole occurs in women of reproductive age with an age ranging from 13 to 49 years. When it occurs in women older than 50 years, it represents a malignant disease [1]. To date, there are few series reported in the world literature of gestational diseases occurring in women older than 50 years. To our knowledge, our case represents one of the rare descriptions in the world literature of a benign complete hydatidiform

Ahmed Samy El-Agwany

.ahmed.elagwany@alexmed.edu.eg

1 Department of Obstetrics and Gynecology, Faculty of Medicine, Alexandria University, Alexandria, Egypt mole in a postmenopausal woman since the first description by Jequier and Winterton in 1973 [2].

\section{Case Report}

A 55-year-old female patient, gravida 7, para 6, and abortion 1 since 15 years with no molar changes was admitted to our department. She was menopausal since 9 years. She was complaining of postmenopausal spotting, and there were no relevant past medical or surgical history. On examination, the uterus was enlarged about 14 weeks of gestation, freely mobile and soft in consistency. Adnexa were not felt with minimal vaginal bleeding on PV examination. Ultrasound examination showed bulky uterus filled with echogenic mass with sonolucent spaces (honeycomb or snowstorm appearance), and uterine walls were intact. There was low resistance flow of uterine arteries $(\mathrm{RI}=0.36)$, and adnexa were free. Gestational trophoblastic tumor was suspected. So, chest radiogram was ordered that showed no pathological lesions of spread. Beta human chorionic gonadotropin assay was $(290,000 \mathrm{mIU} \mathrm{L})$. Laparotomy was done through low transverse incision with exploration of upper abdomen, omentum, intestine, and colon that were free on palpation. Uterus was enlarged, soft, and mobile. Total abdominal hysterectomy with bilateral salpingo-oophorectomy was done for suspicious of malignancy as old age and high titer of $\beta$-hCG. Patient was discharged from the hospital after 2 days with smooth recovery and $\beta$-hCG about $30,324 \mathrm{mIU}$ L. Follow up $\beta$-hCG every week with declining b-hcg that was less than $5 \mathrm{mIU} \mathrm{L}$ on day 27 after surgery. $\beta$-hCG was repeated every week for the next 3 weeks with repeatedly low levels and then was followed every month for 1 year that was undetectable. No prophylactic chemotherapy was required. 
Histopathological examination revealed loosely attached chorionic villi with totally avascular cores and cistern formation. Lining trophoblastic cells (cyto and syncytiotrophoblasts) showed focal hyperplasia with independent solid masses of cells in the background. Mild degenerative atypia was noted. No fetal tissue was present and neither myometrial nor vascular invasion were detected. No malignancy was detected. Ovaries showed well-developed corpus luteum and bilateral corpora albicans. Total abdominal hysterectomy with bilateral salpingo-oophorectomy showed complete (non-invasive) vesicular (hydatidiform) mole.

\section{Discussion}

Gestational trophoblastic disease may be benign or malignant. Benign gestational trophoblastic disease generally occurs in women of reproductive age and is extremely rare in postmenopausal women. In postmenopausal women, it is usually malignant [3]. Molar pregnancy may complicate $1 / 700$ to $1 / 2000$ of pregnancies [4]. Vesicular mole may be complete or partial. Complete mole is more common than partial one with ratio about 3:1 and more recently $1: 1$ [5].

Gestational trophoblastic tumors usually secrete $\beta$-hCG in large amount, and it is found that the serum level is proportional to the volume of the tumor. So, it is easy to be diagnosed but due to its rarity in old age, it is missed or undiagnosed [3]. In patients with complete mole, generalized hydropic degeneration of the chorionic villi with proliferation of trophoblastic cells will be detected and no fetus could be seen in the uterus. Both complete and partial moles can be invasive and invade myometrium, blood vessels, or both. Choriocarcinoma is the malignant form of gestational trophoblastic tumors. Presence of chorionic villi is an important differentiating point between benign and malignant subtypes. Chorionic villi are usually present in molar pregnancies and absent in choriocarcinomas [3]. Also, we can differentiate different types of gestational trophoblastic diseases by the use of immunohistochemical markers as human chorionic gonadotropin, human placental lactogen, P53, and P57 [6, 7].

In 1985, Tsukamoto et al. [8] reported 20 cases of trophoblastic disease in women aged 50 or older. Five patients $(25 \%)$ were diagnosed with choriocarcinoma, seven patients $(35 \%)$ with a hydatidiform mole, and eight patients $(40 \%)$ with invasive mole. However, none of the patients with hydatidiform mole was postmenopausal. In 1997, Davidson et al. [9] described the case of a 60year-old woman diagnosed with a molar pregnancy after only 3 months of amenorrhea. Rabczynski et al. [10] in 2000 reported a case of CHM in a 59-year-old woman; however, the history of amenorrhea was not addressed. In the largest series, Jequier and Winterton [11] reviewed 109 cases of GTD in women older than 50 years. They found malignant disease in $28.4 \%$ (31 patients), benign moles in $47.7 \%$ (52 patients), and an unclear pathologic diagnosis in $23.9 \%$. The age of the patients with benign moles and malignant neoplasms ranged from 50 to 59 years and 51 to 64 years, respectively. There were only two $(1.8 \%)$ postmenopausal patients who had a documented period of amenorrhea greater than 1 year and were diagnosed with a benign mole. These patients were aged 50 and 54 years with amenorrhea for 18 months and 10 years, respectively [11].

In 2004, Garcia et al. described a case of a 61-year-old postmenopausal woman who underwent an emergent total abdominal hysterectomy due to uncontrollable vaginal bleeding associated with an increased serum $\beta$-human chorionic gonadotropin level. The resected uterus contained an endometrial, cystic, and grape-like tumor. Microscopic examination demonstrated hydropic degenerated villi with a circumferential trophoblastic cell proliferation and moderate atypia, consistent with a complete hydatidiform mole [1].

In our case, choriocarcinoma and placental site trophoblastic tumor were ruled out due to the presence of chorionic villi and the absence of tumor necrosis, extreme cytological atypia, or invasion into the myometrium. Partial hydatidiform mole was ruled out owing to the absence of fetal parts or embryo and the presence of a circumferential proliferation of trophoblastic cells in the hydropic chorionic villi. The rarity of a molar pregnancy in this old woman brings the patient's menopausal status into question. Menopause is clinically defined as the period that begins 12 months after the final episode of menstrual bleeding in women. Technically, serumluteinizing hormone and follicle-stimulating hormone levels are increased, and the estradiol levels are decreased. While these laboratory tests may form part of the workup of amenorrhea in the reproductive age, one may not think of checking these serum markers in women older than 50 years with a 1-year history of amenorrhea $[1,2]$.

In our case, we suspected the presence of gestational trophoblastic disease on sonographic examination, and it was confirmed by the high level of serum $\beta$-chug. $\beta$-hCG should be performed in any case with suspected molar changes on ultrasound as in cases of cystic endometrial changes.

\section{Conclusions}

Although molar pregnancy is extremely rare in postmenopausal women, it should be included in the differential 
diagnosis of postmenopausal bleeding especially with ultrasound picture of cystic endometrial changes. We should measure serum level of $\beta$-hCG which is diagnostic.

\section{Compliance with Ethical Standards}

Funding This study was not funded.

Conflict of Interest The authors declare that they have no conflict of interest.

Ethical Approval All procedures performed in studies involving human participants were in accordance with the ethical standards of the institutional and/or national research committee and with the 1964 Helsinki Declaration and its later amendments or comparable ethical standards.

Informed Consent Informed consent was obtained from the patient included in the study.

\section{References}

1. Garcia M et al (2004) Hydatidiform mole in a postmenopausal woman. Arch Pathol Lab Med 128:1039-1042
2. Desai NR et al (2010) Choriocarcinoma in a 73-year-old woman: a case report and review of the literature. J Med Case Rep 4:379

3. Garcia M, Romaguera RL, Gomez-Fernandez C (2004) A hydatidiform mole in a postmenopausal woman. A case report and review of the literature. Arch Pathol Lab Med 128(9):1039 1042

4. Malkwi H, Baraqawi R, Abu-Kheit S, Hiasat M (2003) Molar pregnancy in a 53 years old patient. JRMS 10(2):58-59

5. Fisher RA, Newlands ES (1998) Gestational trophoblastic disease molecular and genetic studies. J Reprod Med 43(1):87-97

6. Castrillon DH, Sun D, Weremowicz S, Fisher RA, Crum CP, Genest DR (2001) Discrimination of complete hydatidiform mole from its mimics by immunohistochemistry of the paternally imprinted gene product p57KIP2. Am J Surg Pathol 25:1225-1230

7. Al-Bozom IA (2000) P53 and Bcl-2 oncoprotein expression in placentas with hydropic changes and partial and complete moles. APMIS 108:756-760

8. Tsukamoto N, Iwasaka T, Kashimura Y, Uchino H, Kashimura M, Matsuyama T (1985) Gestational trophoblastic disease in women aged 50 or more. Gynecol Oncol 20:53-61

9. Davidson SA, Gottesfeld J, La Rosa FG (1997) Molar pregnancy in a 60-year-old woman. Int J Gynecol Obstet 56:53-55

10. Rabczynski J, Kochman A, Prudlak E, Lewandowski M (2000) Hydatidiform mole in 59-year-old woman: a case report. Ginekol Pol 71:152-154

11. Jequier A, Winterton WR (1973) Diagnostic problems of trophoblastic disease in women aged 50 or more. Obstet Gynecol 42:378387 